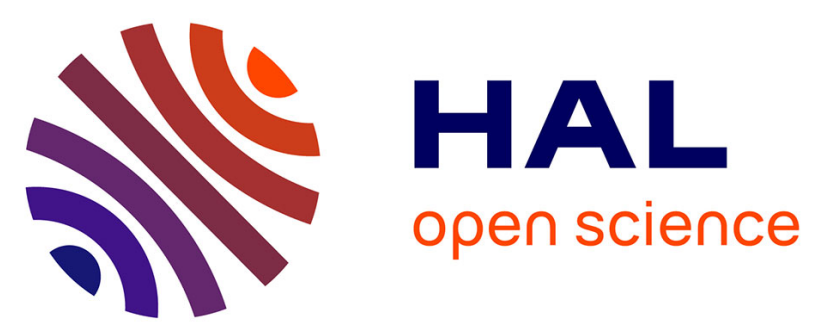

\title{
Comments over homogenisation scales for interfacial emission and scattering by a divided medium: Beerian and non Beerian behaviours
}

Yann Dauvois, Marie Zarrouati, Denis Rochais, Franck Enguehard, Jean Taine

\section{- To cite this version:}

Yann Dauvois, Marie Zarrouati, Denis Rochais, Franck Enguehard, Jean Taine. Comments over homogenisation scales for interfacial emission and scattering by a divided medium: Beerian and non Beerian behaviours. International Journal of Heat and Mass Transfer, 2016, 103, pp.468 - 477. 10.1016/j.ijheatmasstransfer.2016.07.054 . hal-01513607

\section{HAL Id: hal-01513607 \\ https://hal-centralesupelec.archives-ouvertes.fr/hal-01513607}

Submitted on 25 Apr 2017

HAL is a multi-disciplinary open access archive for the deposit and dissemination of scientific research documents, whether they are published or not. The documents may come from teaching and research institutions in France or abroad, or from public or private research centers.
L'archive ouverte pluridisciplinaire HAL, est destinée au dépôt et à la diffusion de documents scientifiques de niveau recherche, publiés ou non, émanant des établissements d'enseignement et de recherche français ou étrangers, des laboratoires publics ou privés. 


\title{
Comments over homogenisation scales for interfacial emission and scattering by a divided medium: Beerian and non Beerian behaviours
}

\author{
Yann Dauvois ${ }^{\mathrm{b}, \mathrm{a}}$, Marie Zarrouatic,a, Denis Rochais ${ }^{\mathrm{b}}$, Franck Enguehard ${ }^{\mathrm{a}}$, Jean \\ Taine $^{\mathrm{a}}$ \\ ${ }^{a}$ CNRS, UPR 288, Laboratoire d'Énergétique Moléculaire et Macroscopique, Combustion (EM2C), \\ CentraleSupelec, Bâtiment Péclet, 92295 Châtenay-Malabry, France. \\ ${ }^{b}$ CEA, DAM, Le Ripault, BP16, 37260 Monts, France. \\ ${ }^{c}$ Air Liquide, Claude et Delorme Research Center, 78350 Les Loges en Josas Cedex, France.
}

\begin{abstract}
In statistical methods of characterisation of porous media radiative properties, interfacial extinction cumulative distribution functions, scattering or absorption cumulative probabilities and general phase functions are generally determined from shots issued from random volume points instead of random interfacial points. Indeed, the first method is numerically much simpler and accurate than the second one. The validity of this approach is discussed and its limitations enhanced for both Beerian and non Beerian homogenised phases, and in the case of a diffuse reflection law or a general one.

The explanation of the identity or difference between the results of the two previous types of extinction cumulative distribution functions comes from the comparison between the spatial scale at which these functions are determined and the own scales of the divided medium. The conditions for which a medium follows the Beer's law are then defined in terms of spatial scales.

Moreover, the modeling of interfacial emission for a non Beerian homogenised phase is in principle based on the reciprocity theorem and an integral formulation of the Generalised Radiative Transfer Equation. The validity of a simpler approach based on an effective absorption coefficient is also discussed, from the previous analysis. The validity of results of recent works published in IJHMT are finally discussed.
\end{abstract}


Keywords: statistical upscaling, interfacial scattering, correlated emission, non Beerian medium, porous medium, non equilibrium radiation.

\section{Nomenclature}

\section{Latin symbols}

n Normal unit vector towards the (semi) transparent phase

$\mathbf{r} \quad$ Coordinates of a current point

$\mathbf{u} \quad$ unit vector of the current direction

C correlation coefficient

E Extinction point

$F \quad$ Radiative distribution function

$f \quad$ Distribution function

$G \quad$ Radiative cumulative distribution function

I Radiative intensity

$M \quad$ Current point

n Refractive index

$P \quad$ Cumulative probability

$S \quad$ Radiative source term

$s, s^{\prime} \quad$ Curvilinear abscissas along a ray

T Temperature

V Volume 
$z \quad$ Axis of the bed $f$ fibers

\section{Greek symbols}

$\kappa \quad$ Absorption coefficient

$\nu \quad$ Frequency

$\alpha \quad$ Interface absorptivity

$\beta \quad$ Extinction coefficient

$\delta \quad$ Kronecker symbol

$\Omega \quad$ Solid angle

$\Phi \quad$ Flux (W)

$\Pi \quad$ Volume fraction or porosity

$\Sigma \quad$ Interfacial area

$\sigma \quad$ Scattering coefficient

\section{Subscripts}

$-{ }_{\nu} \quad$ Frequency

$-{ }_{a} \quad$ Absorption

$-_{\text {ext }}$ Extinction

$-_{e} \quad$ Emission

$-{ }_{o t} \quad$ At optically thin limit

${ }_{-s c} \quad$ Scattering

\section{Superscripts}


$-^{*} \quad$ Conjugated by Fresnel's reflection

-' Related to a volume isotropic source

$-^{\circ} \quad$ At equilibrium

${ }_{-}^{S} \quad$ Related to an interfacial source

\section{Others}

GRTE Generalised Radiative Transfer Equation

OST Medium with Opaque and Semi Transparent phases

OT Medium with Opaque and Transparent phases

RTE Radiative Transfer Equation

ST2 Medium with two Semi Transparent phases

STT Medium with Semi Transparent and Transparent phases

\section{Introduction}

Statistical techniques of characterisation of the radiative properties of homogenised phases of a porous medium based on extinction cumulative distribution functions $G_{e x t \nu}$ and scattering cumulative probabilities $P_{s c \nu}$, have been first developed by Tancrez and Taine [1] and used in many recent works, for media with Opaque and Transparent phases[1, 2, 3, 4, 5, 6] (OT case), with Opaque and Semi Transparent phases $[7,8]$ (OST case), and for media with two Semi Transparent phases or a Semi Transparent phase and a Transparent one[9](ST2 and STT cases).

A perfectly homogeneous and isotropic medium, an absorbing gas for instance, follows the extinction Beer's law: Exponential extinction, characterised by an extinction coefficient $\beta_{\nu}$. A homogenised phase of porous medium can be: i) Strongly non Beerian[1, 4, 7, 10]; It is the general case; ii) Approximately Beerian, as many 
foams[2]; iii) Rigorously Beerian, as the space between statistically homogeneous configurations of overlapping or non overlapping spheres[1] or cylinders[9].

In most of the previous works $G_{\text {ext } \nu}$ is determined by a Statistical Monte Carlo method, based on representative shots issued from random points belonging to volume zones, in all directions[1] for statistically isotropic media, or in elementary solid angles $[2,4,7]$ for statistically anisotropic media, in some cases from surface elements of a phase[11], which are not interfaces between phases. But in the OT case, emission and scattering source terms belong to opaque interfaces; Interfacial source terms also exist in OST case. When is it equivalent to shoot rays from random points within the volume of the real phase or from points of the interfaces? This question is detailed in Sec.2.3. A physical explanation, based on the homogenisation spatial scales, is brought in Sec.3.

When a Beerian model is valid, a precise determination of $G_{e x t \nu}$ and $P_{s c \nu}$ allows accurate values of the extinction and scattering coefficients $\beta_{\nu}$ and $\sigma_{\nu}$ to be obtained $[1,2,3]$, and consequently values of the absorption coefficient $\kappa_{\nu}$. In these conditions, by following the classical approach of radiation transfer, based on Ideal Thermal Equilibrium (ITE) conditions, the emission source term is expressed as $\Pi \kappa_{\nu} n_{\nu}^{2} I_{\nu}^{\circ}(T)$, where $\Pi$ and $n_{\nu}$ are the volume fraction and the refractive index of the homogenised phase. When a homogenised phase is strongly non Beerian, extinction by this phase is exhaustively characterised by $G_{e x t \nu}$ and the scattering source term from $P_{s c \nu}$ and a general scattering phase function $p_{\nu}$. A key question then arises: How to model the emission source term due to an opaque interface in the OT and OST cases, when an absorption coefficient has no more physical meaning? Taine et al.[12] have proposed to use the generalised absorption coefficient at equilibrium $K_{\nu}$, in principle valid for locally optically thick media, and have introduced it in a Generalised Radiative Transfer Equation (GRTE), also directly based on $G_{\text {ext } \nu}$, $P_{s c \nu}$ and $p_{\nu}$. This approach has been applied by Chahlafi et al.[7], but in the particular conditions in which the GRTE degenerates into a classical Beerian RTE, for locally optically thick media: A radiative conductivity tensor has been introduced for characterising degraded rod bundles of a nuclear core in the conditions of a severe accident. 
Zarrouati et al.[11] have recently studied strongly non homogeneous porous media, beds of spheres at the vicinity of a wall, which are strongly non Beerian. A rigorous original model of emission, based on the reciprocity theorem has then been developed. The application of this approach to more common statistically homogeneous phases, that are statistically anisotropic and also strongly non Beerian, is discussed in Sec.4 for the OT and OST cases. The validity conditions of the assumption of Ref.[12] related to emission are defined and a new formulation of the Generalised Radiative Transfer Equation for OT and OST cases is given.

Finally, the validity and the limitations of previously cited studies are discussed in Sec. 5 .

\section{Modeling of radiation issued from interfaces (OT and OST cases)}

This Section first deals with the modeling of radiation issued from an interfacial emission or scattering source term in the OT and OST cases, more precisely with the modeling of the associated extinction cumulative distribution function $G_{e x t}^{S}$, of the scattering or absorption cumulative probability $P_{s c}^{S}$ or $P_{a}^{S}$ and of the scattering phase function $p^{S}[1,2,12,11]$. The superscript ${ }^{S}$ means, here and in the following, that a considered quantity is issued from an interfacial source term. The internal scattering, considered here within a homogenised phase, corresponds in the real porous medium to interfacial reflection. For the sake of simplicity, this work is limited to statistically homogeneous but possibly strongly anisotropic porous media. A case of strongly non homogeneous medium has been studied in Ref.[11].

In the general case, the reflection law is in principle characterised by a bidirectional reflectivity, but the study will be limited for the sake of simplicity and realism, to the case of a Fresnel's law. Emission by opaque interfaces is characterised by an emissivity equal to the absorptivity $\alpha_{\nu}(\mathbf{u . n})$, where $\mathbf{u}$ is the emission direction and $\mathbf{n}(\mathbf{r})$ the normal unit vector at the emission point $M(\mathbf{r})$, oriented towards the (semi) transparent medium.

The radiative statistical functions are built within the real porous medium, from data of tomography samples or from its analytically defined geometry. But, they will be used in the continuous homogenised phase considered as a semi transparent 
medium, in which emission and absorption by interfacial elements become emission and absorption by volume elements, reflection by interfacial elements becomes internal scattering by volume elements.

\subsection{Reflection law (non Beerian homogenised phase)}

In OT case, the extinction cumulative distribution function $G_{e x t}^{S e}\left(\mathbf{u}^{(0)}, s^{\prime}-s\right)$ corresponding to interfacial emission source terms in the direction $\mathbf{u}^{(0)}$ writes for a general interfacial reflection law

$$
\begin{aligned}
& G_{e x t}^{S e}\left(\mathbf{u}^{(0)}, s^{\prime}-s\right)= \\
& \frac{1}{\delta \Omega\left(\mathbf{u}^{(0)}\right)} \int_{s}^{s^{\prime}} \int_{\Sigma / V / \mathbf{u}^{(0)} \cdot \mathbf{n}\left(\mathbf{r}_{0}\right)>0} \int_{\delta \Omega\left(\mathbf{u}^{(0)}\right)} f_{e}^{S}\left[\mathbf{u}^{\prime(0)} \cdot \mathbf{n}\left(\mathbf{r}_{0}\right)\right] \delta\left[s^{\prime \prime}-s_{e x t}\left(\mathbf{r}_{0}, \mathbf{u}^{\prime}(0)\right)\right] \mathrm{d} \Omega\left(\mathbf{u}^{\prime}(0)\right) \mathrm{d} \mathbf{r}_{0} \mathrm{~d} s^{\prime \prime}
\end{aligned}
$$

In Eq.1, the summations are carried out: i) Over all directions $\mathbf{u}^{\prime}{ }^{(0)}$ of an elementary solid angle $\delta \Omega\left(\mathbf{u}^{(0)}\right)$, around the direction $\mathbf{u}^{(0)}$ of the fixed frame; ii) Over all the source points $M_{0}\left(\mathbf{r}_{0}\right)$ of an interfacial surface $\Sigma$ belonging to a volume $V$ of the porous medium such that: $\mathbf{u}^{\prime(0)} \cdot \mathbf{n}\left(\mathbf{r}_{0}\right)>0$. The weight of any current point $M_{0}$ is proportional to $\alpha_{\nu}\left[\mathbf{u}^{\prime(0)} \cdot \mathbf{n}\left(\mathbf{r}_{0}\right)\right]$. Consequently, instead to be weighted by $1 / \Sigma$, the summation over $\mathbf{r}_{0}$ is weighted by the normalised function

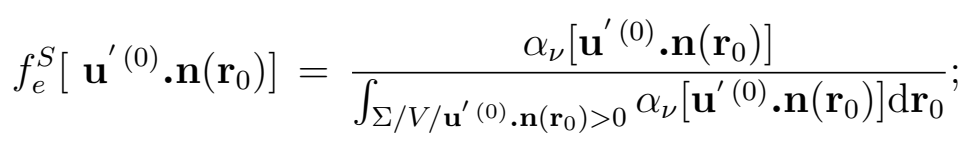

iii) Over all the abscissas $s "$ along an axis of direction $\mathbf{u} ; s_{\text {ext }}\left(\mathbf{r}_{0}, \mathbf{u}^{\prime}{ }^{(0)}\right)$ is the abscissa of the extinction point in the OT case; $\delta$ is the Dirac distribution.

In OST case, Equation 1 is also valid but now the extinction point $E$ can also belong to the semi transparent phase. Its abscissa $s_{e x t}\left(\mathbf{r}_{0}, \mathbf{u}^{\prime}(0), \beta_{\nu}\right)$ is then a point $M$ belonging to the phase volume ( $s_{\text {ext }}$ also depends on the extinction coefficient $\beta_{\nu}$ of this phase) or to an interface.

Moreover, in OST case, an isotropic emission also occurs within the volume of the semi transparent phase. As emission is in this case isotropic, all the volume source points have the same weight. The extinction cumulative distribution function 
$G_{e x t}^{\prime e}\left(\mathbf{u}^{(0)}, s^{\prime}-s\right)$ associated with isotropic volume emission simply writes $G_{e x t}^{\prime e}\left(\mathbf{u}^{(0)}, s^{\prime}-s\right)=\frac{1}{\Pi V} \frac{1}{\delta \Omega\left(\mathbf{u}^{(0)}\right)} \int_{s}^{s^{\prime}} \int_{\Pi V} \int_{\delta \Omega\left(\mathbf{u}^{(0)}\right)} \delta\left[s^{\prime \prime}-s_{e x t}\left(\mathbf{r}_{0}, \mathbf{u}^{\prime}(0), \beta_{\nu}\right)\right] \mathrm{d} \Omega\left(\mathbf{u}^{\prime}(0)\right) \mathrm{d} \mathbf{r}_{0} \mathrm{~d} s^{\prime \prime}$,

where the spatial summation is carried out within all the volume $\Pi V$ of the semi transparent phase, of volume fraction $\Pi$. Note that here and in the following the superscript ' designates any quantity associated with isotropic volume emission.

But, both for OT and OST cases, if the homogenised phase is non Beerian and if a general reflection law or a Fresnel's law is used, the knowledge of the intensity at a point $N$ does not allow the global radiation scattered at this point to be determined[12]. It is due to a memory effect associated with every source term which has partially generated this intensity. More precisely, extinction by scattering of every component of this intensity at the point $N$ has to be correlated with its emission or scattering source term at a point $M$ and with the transmission through the medium from $M$ to $N$.

On the other hand, a scattering source term is considered as non correlated with the original components extinguished by scattering, which have generated it. This assumption is based on the fact that a scattering source term, at a given point in a given direction $\mathbf{u}$, jams the extremely different contributions issued from all the rays extinguished in $4 \pi$ steradians at the same point. Consequently, every set of consecutive internal scattering events can be independently treated. The first set of scattering events corresponds in the real medium to the first reflection phenomena, the second set to the second reflection phenomena, etc. Strongly different extinction cumulative distribution functions are then associated with the successive interfacial scattering source terms corresponding to these sets of scattering events.

The $n^{\text {th }}$ set of interfacial scattering events is characterised at a current interfacial point $M\left(\mathbf{r}_{n}\right)$ of the real medium by $f_{\mathbf{u}^{\prime}(n)}^{S(n)}\left(\mathbf{r}_{n}\right)$, interfacial distribution function of the scattered directions $\mathbf{u}^{\prime(n)}$ in the fixed frame, which depends, at local scale, on the non correlated cumulated incident contributions, of current directions $\mathbf{u}^{\prime(n-1)}$, issued 
from $2 \pi$ steradians. The determination of this quantity is developed in Appendix A. The extinction cumulative distribution function associated with this $n^{\text {th }}$ set of interfacial scattering events is similar to Eq.1, i.e.

$$
\begin{aligned}
& G_{\text {ext }}^{S(n)}\left(\mathbf{u}^{(n)}, s^{\prime}-s\right)= \\
& \int_{s}^{s^{\prime}} \int_{\Sigma^{(n)} / V / \mathbf{u}^{\prime(n)} \cdot \mathbf{n}\left(\mathbf{r}_{n}\right) \geq 0} \int_{\delta \Omega \mathbf{u}^{(n)}} f_{\mathbf{u}^{(n)}}^{S(n)}\left(\mathbf{r}_{n}\right) \delta\left[s^{\prime \prime}-s_{e x t}\left(\mathbf{r}_{\mathbf{n}}, \mathbf{u}^{\prime(n)}, \beta_{\nu}\right)\right] \mathrm{d} \Omega\left(\mathbf{u}^{\prime}(n)\right) \mathrm{d} \mathbf{r}_{n} \mathrm{~d} s^{\prime \prime}
\end{aligned}
$$

In Eq.4, the summations are carried out: i) Over all directions $\mathbf{u}^{\prime}(n)$ belonging to $\delta \Omega \mathbf{u}^{(n)}$; ii) Over all the source points $M\left(\mathbf{r}_{n}\right)$ of the interfacial surface $\Sigma^{(n)}$, part of the interfaces belonging to a volume $V$ of the porous medium which is illuminated by at least one of the $(n-1)^{t h}$ sets of scattering events and contributes to scattering in $\mathrm{d} \Omega\left(\mathbf{u}^{\prime}(n)\right)$. iii) Over all the abscissa $s^{\prime \prime}$ along the axis of direction $\mathbf{u}^{\prime}(n)$. The abscissa of the extinction point $s_{\text {ext }}\left(\mathbf{r}, \mathbf{u}^{\prime}(n), \beta_{\nu}\right)$ belongs to an opaque interface, or possibly to the phase volume in the OST case.

A first distribution function of the scattered directions $f_{\mathbf{u}^{\prime}(1)}^{S(1)}\left(\mathbf{r}_{1}\right)$ and the corresponding extinction cumulative distribution function $G_{\text {ext }}^{S(1)}$ are associated with interfacial emission as source term in both OT and OST cases. In OST case, another first distribution function of the scattered directions $f_{\mathbf{u}^{\prime}(1)}^{\prime}(1)\left(\mathbf{r}_{1}\right)$ and the corresponding extinction cumulative distribution function $G_{\text {ext }}^{\prime s c(1)}$ are associated with isotropic volume emission as source terms. Then, two distribution functions of the scattered directions $f_{\mathbf{u}^{\prime}(2)}^{S(2)}\left(\mathbf{r}_{2}\right)$ and $f_{\mathbf{u}^{\prime}(2)}^{\prime S(2)}\left(\mathbf{r}_{2}\right)$ and the corresponding $G_{\text {ext }}^{S(2)}$ and $G_{e x t}^{\prime S(2)}$ are associated with the second sets of interfacial scattering events; etc. In the general case, all the previously cited $f_{\mathbf{u}^{\prime}(n)}^{S(n)}\left(\mathbf{r}_{n}\right)$ and $f_{\mathbf{u}^{\prime}(1)}^{\prime S(n)}\left(\mathbf{r}_{1}\right)$ and extinction cumulative distribution functions differ. But they are simply determined by a Monte Carlo method, briefly presented in Appendix A.

Some examples of extinction cumulative distribution functions are shown in Fig.1, for a set of overlapping absorbing cylinders of diameter $d=10 \mu m$, length $L=$ $100 D$ and absorption optical thickness $\kappa d=0.3$, in an opaque medium (OST case). The centers of the cylinders have random locations, the unit vectors of their axes $\mathbf{u}_{C}\left(\theta_{C}, \phi_{C}\right)$ are characterised by random values of $\phi_{C}$ and a Gaussian distribution of $\theta_{C}$ centered in the plane normal to the $z$ axis, with a standard deviation equal 

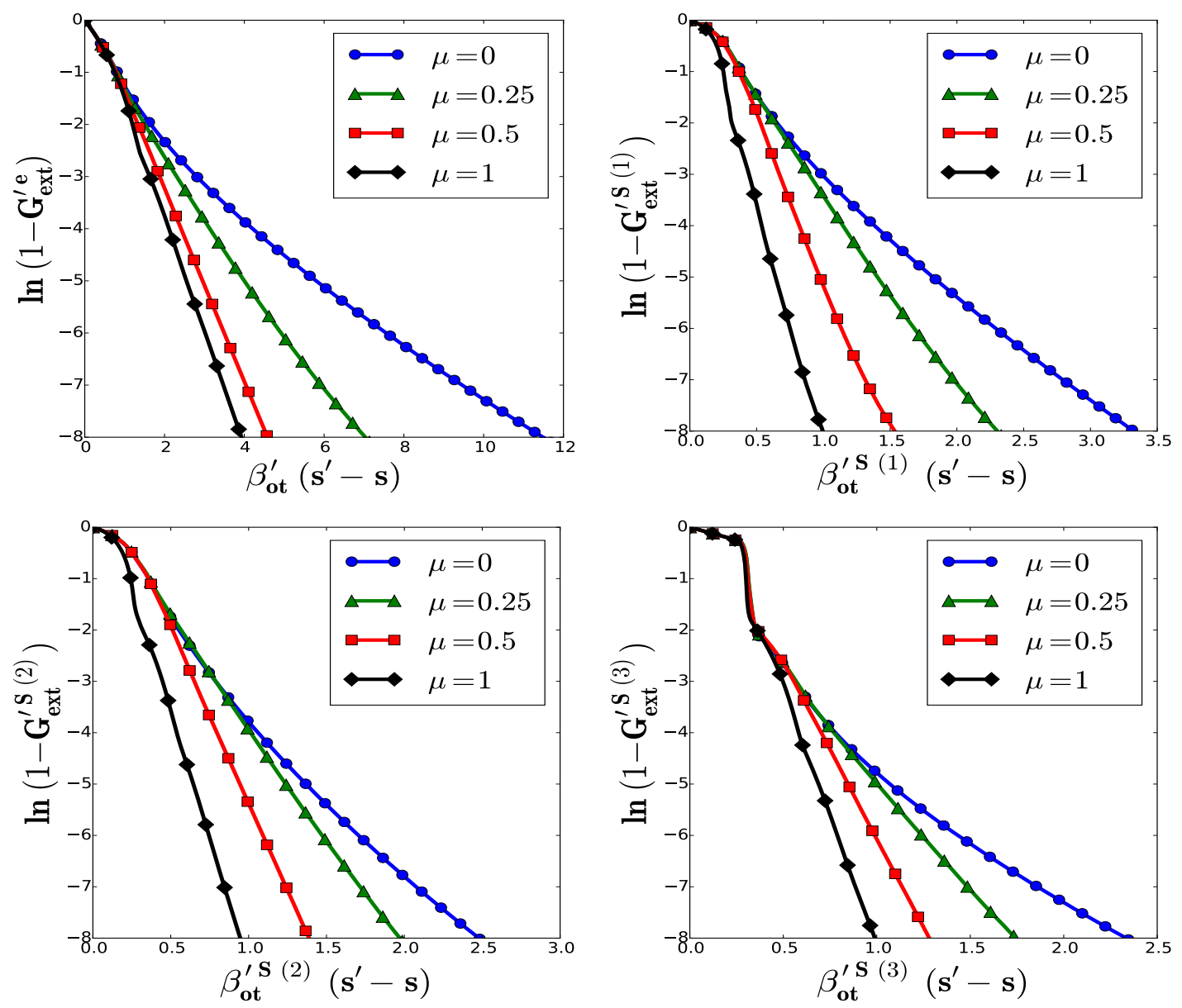

Figure 1: Extinction cumulative distribution functions associated with semi transparent overlapping cylinders within an opaque phase (OST non Beerian case). In all cases: Upper left figure: $G_{e x t}^{\prime e}$ issued from random volume isotropic emission points; Upper right figure: $G_{e x t}^{\prime} S^{(1)}$ associated with the first set of internally scattered rays; Lower left figure: $G_{e x t}^{\prime S(2)}$ associated with the second set of internally scattered rays; Lower right figure: $G_{e x t}^{\prime S(3)}$ associated with the third set of internally scattered rays; $\kappa_{\nu} d=0.3$.

to $3^{\circ}$. The medium is statistically homogeneous, statistically isotropic in any plane perpendicular to the $z$ axis, but strongly anisotropic in other directions.

In the following, all the extinction cumulative distribution functions are plotted vs $\beta_{o t}\left(s^{\prime}-s\right)$, where $\beta_{o t}$ is the extinction coefficient at the optically thin limit determined 
by the derivative of these functions at the origin, i.e.

$$
\beta_{o t}=\frac{\mathrm{d} G_{e x t}}{\mathrm{~d} s^{\prime}}\left(\mathbf{u}, s^{\prime}-s=0\right)
$$

Note that the values of $\beta_{o t}$ differ for the different extinction cumulative distribution functions.

Consider first $G_{\text {ext }}^{\prime e}(\mu)$ associated with isotropic volume emission source terms and where $\mu$ is the director cosine of the direction $\mathbf{u}^{(0)}$ vs the $z$ axis. $G_{e x t}^{\prime e}(\mu)$ has been determined from a huge number of shots issued from random volume emission points within the semi transparent propagation phase in an elementary solid angle $\delta \Omega$. The homogenised phase is strongly non Beerian as shown in upper left Fig.1: Indeed, $\ln \left(1-G_{e x t}^{\prime e}\right)$ strongly deviates from a straight line for any $\mu$ value.

In a second step, $G_{\text {ext }}^{\prime S(1)}(\mu)$ has been determined by using the first set of reflected rays, issued from the initial rays emitted within the semi transparent phase volume. An arbitrary reflection law is defined by the Fresnel's law corresponding to a value $n_{o} / n_{p}=2$ of the ratio of the refractive index of the opaque phase to the one of the propagation phase. As shown in upper right Fig.1, $G_{e x t}^{\prime S(1)}(\mu)$ strongly differs from $G_{\text {ext }}^{\prime e}(\mu)$.

In a third step, $G_{\text {ext }}^{\prime S(2)}(\mu)$ and $G_{\text {ext }}^{\prime S}(3)(\mu)$ have been determined by using the second and third sets of reflected rays, issued from the first and second sets of reflected ones, respectively. As shown in the two lower Figs.1, $G_{e x t}^{\prime e}(\mu), G_{e x t}^{\prime S(1)}(\mu), G_{e x t}^{\prime S(2)}(\mu)$ and $G_{\text {ext }}^{\prime S(3)}(\mu)$ all strongly differ.

The corresponding probabilities of absorption $\mathcal{P}_{a \nu}^{\left({ }^{\prime}\right)} S(n)\left(\mathbf{u}^{(n)}\right)$ and scattering $\mathcal{P}_{\text {sc }}^{\left({ }^{\prime}\right)}{ }^{(n)}\left(\mathbf{u}^{(n)}\right)$ are determined in parallel with the general phase functions $p_{\nu n}^{\left({ }^{\prime}\right) S}{ }^{(n)}\left(\mathbf{u}^{(n)}, \mathbf{u}^{(n+1)}\right)$. The scattering cumulative probability $P_{s c \nu}^{\left({ }^{\prime}\right) S(n)}\left(\mathbf{u}^{(n)}, s^{\prime}-s\right)$ is then equal to $\mathcal{P}_{s c}^{\left({ }^{\prime}\right) S(n)}\left(\mathbf{u}^{(n)}\right)$ $G_{\text {ext }}^{\left({ }^{\prime}\right) S s c(n)}\left(\mathbf{u}^{(n)}, s^{\prime}-s\right)$ and the absorption cumulative probability $P_{a \nu}^{\left({ }^{\prime}\right) S(n)}\left(\mathbf{u}^{(n)}, s^{\prime}-s\right)$ to $\mathcal{P}_{a}^{\left({ }^{\prime}\right) S(n)}\left(\mathbf{u}^{(n)}\right) G_{\text {ext }}^{\left({ }^{\prime}\right) S s c(n)}\left(\mathbf{u}^{(n)}, s^{\prime}-s\right)$.

If we account for the system symmetries the phase function depends in principle on $\mu$ director cosine of the incidence direction, on $\mu_{s c}$ director cosine of the internally scattered one and on the azimuth difference $\phi_{s c}-\phi$. As this last dependence is 

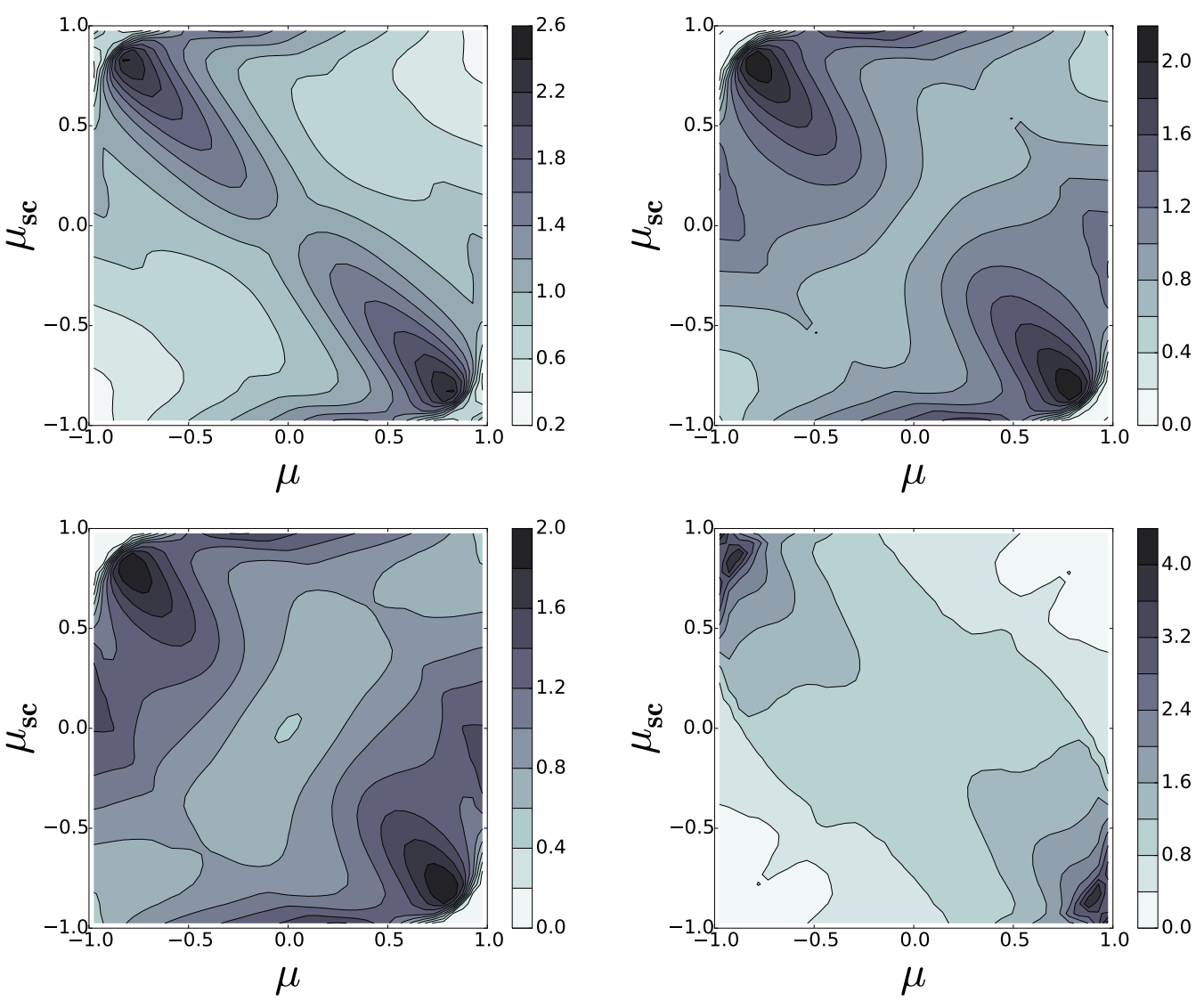

Figure 2: Averaged phase functions associated with semi transparent overlapping cylinders within an opaque phase (OST non Beerian case); Upper left: $p^{\prime} e\left(\mu, \mu_{s c}\right)$ issued from random volume isotropic emission points; Upper right: $p^{\prime} S^{(1)}\left(\mu, \mu_{s c}\right)$ associated with the first set of internally scattered rays; Lower left: $p^{\prime S(2)}\left(\mu, \mu_{s c}\right)$ associated with the second set of internally scattered rays; Lower right: $p^{\prime}{ }^{(3)}\left(\mu, \mu_{s c}\right)$ associated with the third set of internally scattered rays; $\kappa_{\nu} d=0.3$.

of weak interest for radiative transfer, the phase functions have been averaged over $\phi_{s c}-\phi$ values. The averaged phase functions associated with incident rays issued from the isotropic volume emission points and from the first, second and third sets of scattered rays also strongly differ, as shown in Fig.2. 


\subsection{Diffuse reflection (non Beerian homogenised phase)}

Consider now, in OT case, the popular assumption of a diffuse reflection law at the interfaces, for a general non Beerian homogenised phase. In these conditions, the scattered local intensity is isotropic as the emitted local intensity. Moreover, emitted and scattered rays are characterised by the same distribution function $f^{S}$ which simply is a scalar. Consequently, the modeling of the extinction cumulative distribution functions is strongly simplified.

The unique extinction cumulative distribution function $G_{\text {ext }}^{S}\left(\mathbf{u}, s^{\prime}-s\right)$ of the homogenised phase associated with diffuse interfacial elements then writes in OT case, for both emission and scattering source terms,

$$
G_{e x t}^{S}\left(\mathbf{u}, s^{\prime}-s\right)=\frac{1}{\delta \Sigma} \frac{1}{\delta \Omega} \int_{s}^{s^{\prime}} \int_{\delta \Omega / \mathbf{u} . \mathbf{n}<0} \int_{\delta \Sigma / V} \delta\left[s^{\prime \prime}-s_{e x t}(\mathbf{r}, \mathbf{u}] \mathrm{d} \mathbf{r} \mathrm{d} \Omega(\mathbf{u}) \mathrm{d} s^{\prime \prime},\right.
$$

where $s^{\prime}-s$ is in fact the length of the chord between the current interfacial source point $M(s)$ and the current interfacial extinction point $E\left(s^{\prime}\right) . G_{e x t}^{S}\left(\mathbf{u}, s^{\prime}-s\right)$ is interpreted as the cumulative distribution function of the chords $M E$ of length $s^{\prime}-s$; As $G_{e x t}^{S}\left(\mathbf{u}, s^{\prime}-s\right)$ is then a purely geometrical quantity, it does not depend on frequency. More commonly, $G_{e x t}^{S}\left(\mathbf{u}, s^{\prime}-s\right)$ is also equal to $1-\tau^{S}\left(\mathbf{u}, s^{\prime}-s\right)$, where $\tau^{S}\left(\mathbf{u}, s^{\prime}-s\right)$ is the transmissivity from $s$ to $s^{\prime}$ in direction $\mathbf{u}$.

Similarly, $P_{s c \nu}^{S}\left(\mathbf{u}, s^{\prime}-s\right)$, or $P_{a \nu}^{S}\left(\mathbf{u}, s^{\prime}-s\right)$, is the probability that a radiation issued from an interfacial emission or scattering source point $M(s)$ is scattered, or absorbed, by an interface before the distance $s^{\prime}-s$. For the considered diffuse reflection law characterised by an absorptivity $\alpha_{\nu}, P_{s c \nu}^{S}\left(\mathbf{u}, s^{\prime}-s\right)$ is simply equal to $\left(1-\alpha_{\nu}\right) G_{e x t}^{S}\left(\mathbf{u}, s^{\prime}-s\right)$ and $P_{a \nu}^{S}\left(\mathbf{u}, s^{\prime}-s\right)$ to $\alpha_{\nu} G_{e x t}^{S}\left(\mathbf{u}, s^{\prime}-s\right)$.

From a mathematical point of view, $1-G_{e x t}^{S}\left(\mathbf{u}, s^{\prime}-s\right)$ characterising a diffuse reflection law, transmissivity from $s$ to $s^{\prime}$ in the direction $\mathbf{u}$ in the homogenised phase, is proportional to the distribution function $F_{e x t}^{\prime}\left(\mathbf{u}, s^{\prime}-s\right)$ of the segments $M E$, now joining a current phase volume point $M(s)$ to the current extinction point $E\left(s^{\prime}\right)$ in a given direction $\mathbf{u}$. Remember that a quantity associated with volume isotropic source points, such as $F_{e x t}^{\prime}$, presents a superscript ' instead of ${ }^{S} . F_{e x t}^{\prime}\left(\mathbf{u}, s^{\prime}-s\right)$ is in fact the derivative of $G_{e x t}^{\prime}\left(\mathbf{u}, s^{\prime}-s\right)$, corresponding to the extinction cumulative distribution 
function associated with volume isotropic source points. As the transmissivity $1-$ $G_{e x t}^{S}\left(\mathbf{u}, s^{\prime}-s\right)$ belongs to the range [0,1], $G_{e x t}^{S}\left(\mathbf{u}, s^{\prime}-s\right)$ is equal to

$$
G_{e x t}^{S}\left(\mathbf{u}, s^{\prime}-s\right)=1-\frac{F_{e x t}^{\prime}\left(\mathbf{u}, s^{\prime}-s\right)}{F_{e x t}^{\prime}(\mathbf{u}, 0)} .
$$

$G_{e x t}^{S}$ associated with a diffuse reflection law is finally linked to $G_{e x t}^{\prime}$ by

$$
G_{e x t}^{S}\left(\mathbf{u}, s^{\prime}-s\right)=1-\left(\frac{\mathrm{d}}{\mathrm{d} s^{\prime}} G_{e x t}^{\prime}\left(\mathbf{u}, s^{\prime}-s\right) / \frac{\mathrm{d}}{\mathrm{d} s^{\prime}} G_{e x t}^{\prime}(\mathbf{u}, 0)\right) .
$$

Equation 8 is valid for any non Beerian homogenised phase in the OT case, when the opaque phase is characterised by a diffuse reflection law.

In a medium with semi transparent and opaque phases (OST case), extinction by the real Beerian semi transparent phase is statistically independent of extinction by the homogenised non Beerian phase associated with interfacial elements. Consequently, the global transmissivity of the medium simply is the product of the transmissivities, i.e.

$$
G_{e x t \nu}^{S}\left(\mathbf{u}, s^{\prime}-s\right)=1-\left(\exp \left[-\beta_{\nu}\left(s^{\prime}-s\right)\right]\left[1-G_{e x t}^{S}\left(\mathbf{u}, s^{\prime}-s\right)\right]\right),
$$

where $G_{e x t t}^{S}\left(\mathbf{u}, s^{\prime}-s\right)$ is the extinction cumulative distribution function of the corresponding porous medium with a transparent phase (OT case).

\subsection{Beerian homogenised phase (All types of reflection)}

Consider first a medium with a transparent and an opaque phase, characterised by a diffuse reflection law. It is now assumed that $G_{e x t}^{\prime}\left(\mathbf{u}, s^{\prime}-s\right)$, the cumulative extinction distribution function associated with random isotropic source points (for instance emission points) within the transparent phase is Beerian, i.e. equal to $1-\exp \left[-\beta(\mathbf{u})\left(s^{\prime}-s\right)\right]$, where $\beta(\mathbf{u})$ is an extinction coefficient, a priori depending on the direction $\mathbf{u}$. From Eq.8, $G_{e x t}^{S}\left(\mathbf{u}, s^{\prime}-s\right)$ is then also equal to $G_{e x t}^{\prime}\left(\mathbf{u}, s^{\prime}-s\right)$. In these conditions, both for a scattering or an emission source term in the direction $\mathbf{u}$, the interfacial cumulative distribution function associated with a diffuse reflection law then simply writes in the OT case

$$
G_{e x t}^{S}\left(\mathbf{u}, s^{\prime}-s\right)=G_{e x t}^{\prime}\left(\mathbf{u}, s^{\prime}-s\right)=1-\exp \left[-\beta(\mathbf{u})\left(s^{\prime}-s\right)\right] .
$$


For a given direction $\mathbf{u}$, the same distribution function of the lengths characterises both the chords between interfacial points and the segments between volume points and interfacial points. In practice, $G_{e x t}^{\prime}\left(\mathbf{u}, s^{\prime}-s\right)$ is generally used instead of $G_{e x t}^{S}\left(\mathbf{u}, s^{\prime}-\right.$ $s$ ), as for instance in left Fig.3, because its determination is simpler and more accurate.
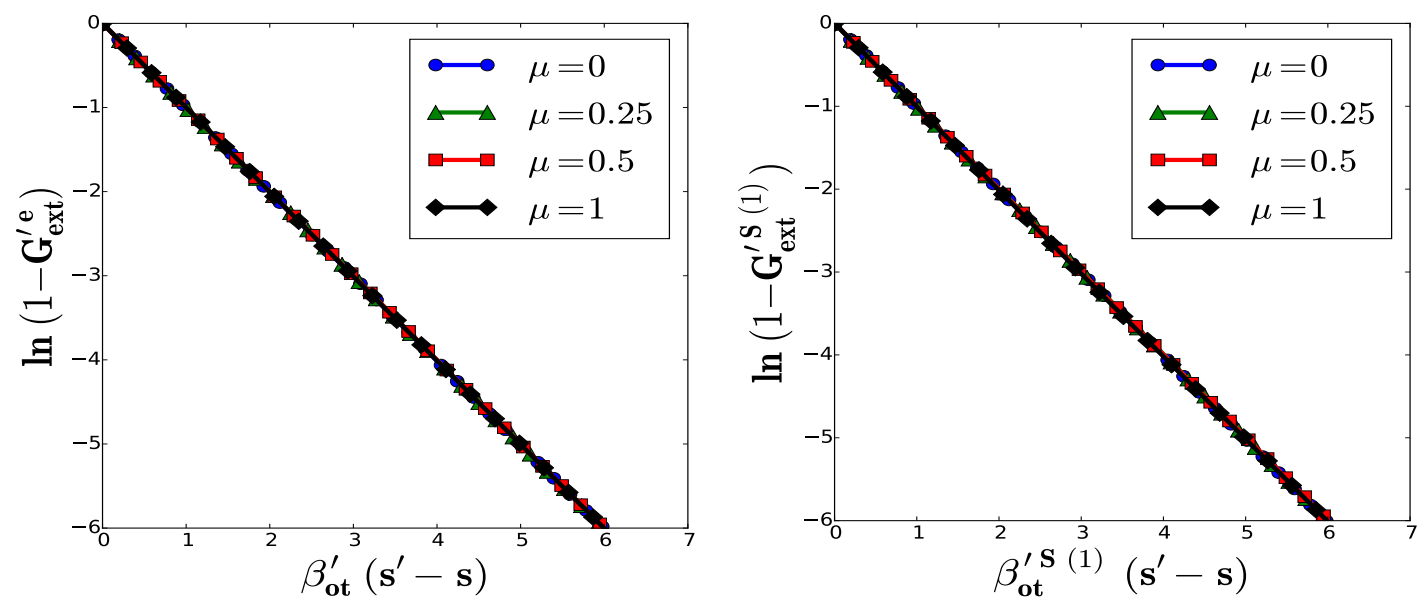

Figure 3: Extinction cumulative distribution functions associated with the transparent propagation phase between overlapping opaque cylinders (OT Beerian case); Left: $G_{\text {ext }}^{\prime}$ issued from random isotropic volume points, equal to $G_{e x t}^{S}\left(\mathbf{u}, s^{\prime}-s\right)$ associated with a diffuse reflection law; Right: $G_{\text {ext }}^{\prime S(1)}$ associated with the first set of scattered rays following a Fresnel's law.

The same medium is now assumed characterised by a Fresnel's reflection law. The right Figure 3 compares, for instance, the extinction cumulative distribution function $G_{e x t}^{\prime S(1)}$ associated with the first set of scattered rays following a Fresnel's law with the previous result $G_{e x t}^{\prime}\left(\mathbf{u}, s^{\prime}-s\right)$. Note that the extinction coefficients at the optically thin limit associated with the two extinction distribution functions $\beta_{o t}^{\prime S(1)}$ and $\beta_{o t}^{\prime}$ are exactly equal. As the normalised functions $\ln \left[1-G_{e x t}^{\prime S(1)}\right]$ and $\ln \left[1-G_{e x t}^{\prime}\left(\mathbf{u}, s^{\prime}-s\right)\right]$ are identical, the same extinction coefficient $\beta(\mu)$, also equal to $\beta_{o t}^{\prime S(1)}$ and $\beta_{o t}^{\prime}$, exhaustively characterises the two whole extinction cumulative distribution functions, which are Beerian.

This property is general, for a Beerian homogenised phase in the OT case, whatever the reflection law at the interfaces: The extinction cumulative distribution function 
associated with any set $(n)$ of scattered rays is Beerian and, in practice, determined from random isotropic volume points within the phase. A physical explanation of this property is given in Sec.3.

An important consequence is that, for any Beerian homogenised phase, the phase functions $p_{\nu}^{\prime S(1)}\left(\mu, \mu_{r}\right)$, associated with the previously defined specular interfacial source terms are then identical to $p_{\nu}\left(\mathbf{u}_{1}, \mathbf{u}\right)$, associated with isotropic volume source terms. This is numerically shown in Fig.4. The very small differences between the two phase functions are due to numerical errors.
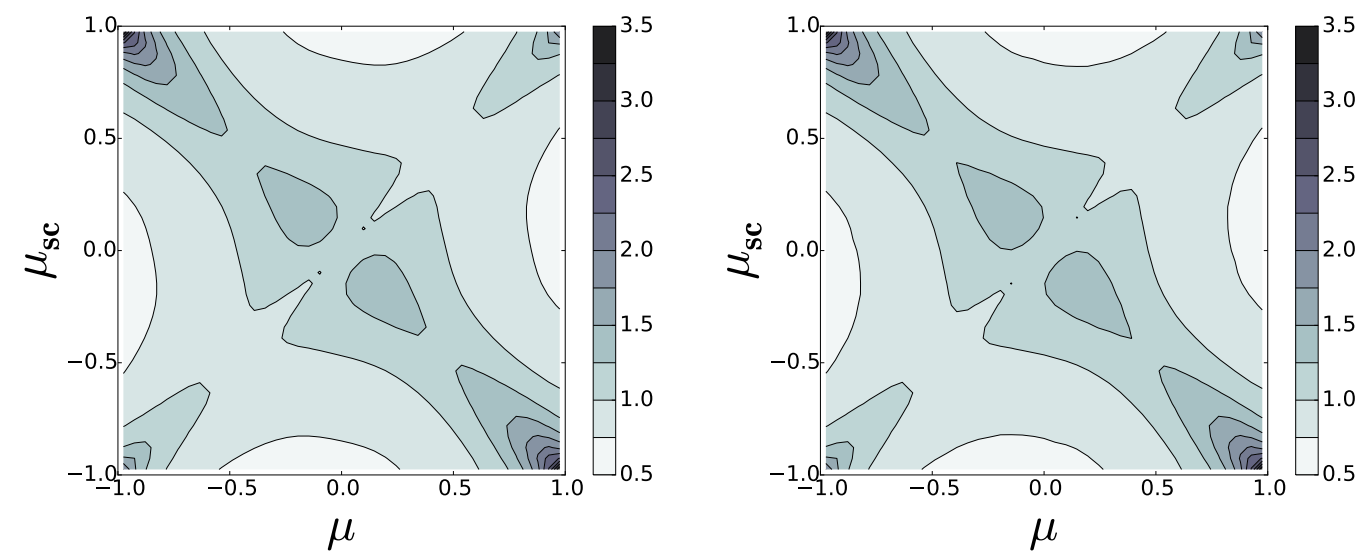

Figure 4: Phase functions associated with a transparent propagation phase between overlapping opaque cylinders (OT Beerian case); Left: $p\left(\mu, \mu_{r}\right)$ issued from random volume points; Right: $p^{\prime} S^{(1)}\left(\mu, \mu_{r}\right)$ associated with the first set of scattered rays following a Fresnel's law.

\section{Homogenisation scale and Beerian model scale}

In the statistical approach, an extinction cumulative distribution function of a non Beerian homogenised phase, which is statistically homogeneous and possibly strongly anisotropic, is determined from volume or interfacial points issued from a shooting zone representative of the medium (see for instance Refs.[1], [7]). The phase is in fact statistically homogeneous at a spatial scale a priori larger than the typical size $\Delta$ of the shooting zone. This zone is generally defined by using a possible system periodicity and a perfect specular reflection at its boundaries[7], or by checking the reproducibility with results of other shooting zones[2], or simply by numerical 
building[1] in the case of a medium model. An extinction zone, which is generally larger than the shooting zone, has a size $D$, chosen in such a way that practically all rays are extinguished in any direction within the extinction zone. The material structure is strongly non homogeneous at a scale a priori smaller than $D$, for a given direction $\mathbf{u}$.

From the study of the previous Section, it appears that for a non Beerian homogenised phase, the extinction cumulative distribution functions $G_{e x t}^{S}\left(\mathbf{u}, s^{\prime}-s\right)$ associated with emission and interfacial scattering strongly depend on two types of distribution functions: i) On the distribution function of u.n, associated with the emission law at the interfaces; ii) On the distribution function of the scattered directions at these source points, which itself depends on the extinction within a possible semi transparent medium or on previous scattering events.

Extinction phenomena within an interval $\left[s^{\prime}, s^{\prime}+\mathrm{d} s^{\prime}\right]$ due to emission and scattering source terms within $[s, s+\mathrm{d} s]$, are then strongly correlated with the two previous distribution functions and with the specific scattering and absorption events between $s$ and $s^{\prime}$ associated with the strong non homogeneity of the medium at a scale smaller than $D$. A previously cited consequence of any type of these correlations is that the global intensity at the point $s^{\prime}$ does not bring a sufficient information for determining the global extinction within $\left[s^{\prime}, s^{\prime}+\mathrm{d} s^{\prime}\right]$. This is due to the fact that the transmissivity between $s$ and $s^{\prime}$ is strongly correlated with extinction within $\left[s^{\prime}, s^{\prime}+\mathrm{d} s^{\prime}\right]$ but also with a previous type of distribution function. The successive extinction distribution functions associated with interfacial sources $G_{e x t}^{S(n)}\left(\mathbf{u}, s^{\prime}-s\right)$ introduced in Sec.2.3 account for all these types of correlations.

For a non Beerian homogenised phase, $G_{e x t}\left(\mathbf{u}, s^{\prime}-s\right)$ is associated with an isotropic volume source term, typically by emission: There is then only one correlation between transmission from the source point $s$ to $s^{\prime}$ and extinction in the range $\left[s^{\prime}, s^{\prime}+\mathrm{d} s^{\prime}\right]$. Similarly, when $G_{e x t}^{S}\left(\mathbf{u}, s^{\prime}-s\right)$, associated with interfacial emission or scattering, also corresponds to a local diffuse reflection law, the same unique previous correlation also occurs. In these particular cases, the extinction cumulative distribution functions $G_{e x t}\left(\mathbf{u}, s^{\prime}-s\right)$ and $G_{e x t}^{S}\left(\mathbf{u}, s^{\prime}-s\right)$ obviously differ but are rigorously linked 
by Eq.8.

If the homogenisation scale of a non Beerian extinction cumulative distribution function is $D$, larger than $\Delta$, the homogenisation scale of a Beerian medium is arbitrarily small compared to $D$. Indeed any element $\left[s^{\prime \prime}, s^{\prime \prime}+\mathrm{d} s^{\prime \prime}\right]$ of the interval $\left[s, s^{\prime}\right]$ has exactly the same statistical universal properties as any other element, $[s, s+\mathrm{d} s]$ or $\left[s^{\prime}+\mathrm{d} s^{\prime}\right]$ for instance. More precisely the transmissivity from $s$ to $s^{\prime}, 1-G_{\text {ext }}\left(\mathbf{u}, s^{\prime}-s\right)$ is non correlated with the source term in the range $[s, s+\mathrm{d} s]$ and with the extinction term in $\left[s^{\prime}, s^{\prime}+\mathrm{d} s^{\prime}\right]$. In these conditions of non correlation in $s^{\prime}$, the variation $\mathrm{d} I_{\nu}\left(\mathbf{u}, s^{\prime}\right)$ of the intensity $I_{\nu}\left(\mathbf{u}, s^{\prime}\right)$ between $s^{\prime}$ and $s^{\prime}+\mathrm{d} s^{\prime}$ is proportional to $I_{\nu}\left(\mathbf{u}, s^{\prime}\right)$, which is typical of an exponential Beerian function, characterised by an extinction coefficient $\beta(\mathbf{u})$.

The remarkable non correlation property between a source term within $[s, s+\mathrm{d} s]$ and the transmission between $s$ and $s^{\prime}$ explains that the transmissivity and consequently $G_{\text {ext }}^{S}\left(\mathbf{u}, s^{\prime}-s\right)$ are independent of the distribution function of $\mathbf{u . n}$, for an emission source, and of the distribution function of the scattered directions, for a scattering source. $G_{\text {ext }}^{S}\left(\mathbf{u}, s^{\prime}-s\right)$ is then equal to $G_{\text {ext }}\left(\mathbf{u}, s^{\prime}-s\right)$.

If the validity conditions of the radiative Fourier's law[13] are fulfilled, i.e. when the medium is quasi isothermal at the scale of $D$, a radiative transfer only occurs at a spatial scale larger than $D$. The discretised elements $[s ", s "+\mathrm{d} s "]$ of the previous analysis have now sizes larger than $D$ and are non correlated. In these conditions, the homogenised phase is Beerian at this large scale and all the previous results are valid.

\section{Correlated modeling of interfacial emission}

As previously discussed, the modeling of interfacial scattering is based on the assumption of a non global correlation between the incidence radiation distribution and the scattered radiation. On the contrary, a strong correlation appears between emission and transmission and has to be accounted for in the modeling of interfacial emission within non Beerian homogenised phases in the OT and OST cases. 
The notations of Sec.2 are used in the following. If the homogenised phase is Beerian, it is simply characterised by an absorption coefficient $\kappa_{\nu}^{S}$. The emission source term of this phase has then the same expression as for a real semi transparent phase $[14,15,16]$

$$
S_{e \nu}^{S}(\mathbf{u}, s)=\Pi \kappa_{\nu}^{S} n_{\nu}^{2} I_{\nu}^{\circ}\left[T_{i}(s)\right] .
$$

where $\Pi$ and $n_{\nu}$ respectively are the volume fraction and the refractive index of the transparent or semi transparent propagation phase; $T_{i}(s)$ is the interfacial temperature field, which a priori differs from the opaque phase temperature field.

If this phase is non Beerian, an absorption coefficient has no more physical meaning in the general case. The extinction by absorption or scattering within this phase is accurately modeled by the absorption and scattering cumulative probabilities $P_{a \nu}$ and $P_{s c \nu}$ instead of absorption and scattering coefficients [12]. But, the modeling of emission within the phase is much less obvious. In the classical theory of thermal radiation a similar difficulty is solved by the assumption that, for Beerian media, the emission source term is the same as in Local Thermal Equilibrium (LTE) [14, 15, 16]. By analogy with this approach, Taine et al.[12] have assumed that the emission term of a non Beerian phase is the same as in LTE conditions, i.e. given by Eq.11 in which $K_{\nu}$, generalised absorption coefficient at equilibrium defined in Ref.[12], is used. The weakness of this approach has been highlighted by Zarrouati et al.[11] for statistically non homogeneous phases: Along a ray within the non Beerian phase, emission between $s$ and $s+\mathrm{d} s$ is strongly correlated to transmission from $s$ to $s^{\prime}$, exactly as transmission from $s$ to $s^{\prime}$ is strongly correlated to extinction by absorption and scattering between $s^{\prime}$ and $s^{\prime}+\mathrm{d} s^{\prime}$. It is also the case for any non Beerian statistically homogeneous phase. More precisely, the weakness of the previous assumption of Ref[12] is that emission is non correlated to transmission.

As, in the model, transmission from $s$ to $s^{\prime}$ is accurately correlated to absorption between $s^{\prime}$ and $s^{\prime}+\mathrm{d} s^{\prime}$, a simple idea for modeling the correlation between emission and transmission is to use the reciprocity theorem. This approach, developed for a non homogeneous phase in Ref.[11], is here briefly summarised for a statistically homogeneous phase and is valid for porous media with an opaque phase (OT and 
OST cases).

The power emitted by a black body of temperature $T_{b}$ in the elementary solid angle $\mathrm{d} \Omega(-\mathbf{u})$ at a point $M^{\prime}\left(s^{\prime}\right)$, transmitted from $s^{\prime}$ to $s$ and absorbed by interfacial elements belonging to a volume element around the point $M(s)$, by unit volume, unit frequency and unit solid angle, writes

$$
\frac{\mathrm{d}^{6} \Phi^{S s^{\prime} s}}{\mathrm{~d} V \mathrm{~d} \Omega \mathrm{d} \nu}\left(-\mathbf{u}, s-s^{\prime}\right)=\Pi \frac{\mathrm{d}}{\mathrm{d} s} P_{a \nu}^{S}\left(-\mathbf{u}, s-s^{\prime}\right) n_{\nu}^{2} I_{\nu}^{\circ}\left(T_{b}\right)
$$

where $P_{a \nu}^{S}$ is the absorption cumulative probability from $s^{\prime}$ to $s$, or from $s$ to $s^{\prime}$. According to the reciprocity theorem, the power emitted by the same interfacial elements of temperature $T_{i}(s)$ belonging to a volume element around the point $M(s)$, in the elementary solid angle $\mathrm{d} \Omega(\mathbf{u})$, by unit volume, unit frequency and unit solid angle, transmitted from $s$ to $s^{\prime}$ and absorbed by the black body around $s^{\prime}$, writes

$$
\frac{\mathrm{d}^{6} \Phi_{\text {e } s^{\prime}}^{S s_{\nu}}}{\mathrm{d} V \mathrm{~d} \Omega \mathrm{d} \nu}\left(\mathbf{u}, s^{\prime}-s\right)=\frac{\mathrm{d}^{6} \Phi_{\text {e }}^{S s^{\prime} s}}{\mathrm{~d} V \mathrm{~d} \Omega \mathrm{d} \nu}\left(-\mathbf{u}, s-s^{\prime}\right)\left(\frac{I_{\nu}^{\circ}\left[T_{i}(s)\right]}{I_{\nu}^{\circ}\left(T_{b}\right)}\right)=\Pi \frac{\mathrm{d}}{\mathrm{d} s} P_{a \nu}^{S}\left(\mathbf{u}, s^{\prime}-s\right) n_{\nu}^{2} I_{\nu}^{\circ}\left[T_{i}(s)\right],
$$

where $P_{a \nu}^{S}\left(\mathbf{u}, s^{\prime}-s\right)$ is equal to $P_{a \nu}^{S}\left(-\mathbf{u}, s-s^{\prime}\right)$, due to its invariance by time reversing. Finally, the emission source term due to the interfacial opaque elements belonging to a volume element $\mathrm{d} V$ around $s$ by unit volume, unit solid angle and unit frequency, correlated with transmission from $s$ to $s^{\prime}$, writes

$\overline{S_{e \nu}^{S}(\mathbf{u}, s) \tau_{\nu}\left(\mathbf{u}, s^{\prime}-s\right)}=\overline{S_{e \nu}^{S}(\mathbf{u}, s)\left[1-G_{e x t}\left(\mathbf{u}, s^{\prime}-s\right)\right]}=\Pi \frac{\mathrm{d}}{\mathrm{d} s} P_{a \nu}^{S}\left(\mathbf{u}, s^{\prime}-s\right) n_{\nu}^{2} I_{\nu}^{\circ}\left[T_{i}(s)\right]$.

Note that, if the homogenised phase is Beerian[1]

$$
\frac{\mathrm{d}}{\mathrm{d} s} P_{a \nu}^{S}\left(\mathbf{u}, s^{\prime}-s\right)=\kappa_{\nu}(\mathbf{u})\left[1-G_{e x t}\left(\mathbf{u}, s^{\prime}-s\right)\right]=\kappa_{\nu}(\mathbf{u}) \exp \left[-\beta_{\nu}(\mathbf{u})\left(s^{\prime}-s\right)\right]
$$

the emission source term is not correlated with the transmission from $s$ to $s^{\prime}$ and the result given by Eq.14 is consistent with Eq.11. A correlation coefficient is then defined[11] by

$$
C\left(\mathbf{u}, s^{\prime}-s\right)=\frac{\mathrm{d}}{\mathrm{d} s} P_{a \nu}^{S}\left(\mathbf{u}, s^{\prime}-s\right) /\left(\frac{\mathrm{d}}{\mathrm{d} s} P_{a \nu}^{S}(\mathbf{u}, 0)\left[1-G_{e x t \nu}^{S}\left(\mathbf{u}, s^{\prime}-s\right)\right]\right) .
$$


Its determination allows the emission source term correlated with transmission from $s$ to $s^{\prime}$ to be written

$$
\overline{S_{e \nu}^{S}(\mathbf{u}, s) \tau_{\nu}\left(\mathbf{u}, s^{\prime}-s\right)}=\left(C\left(\mathbf{u}, s^{\prime}-s\right) \kappa_{\nu o t}^{S}(\mathbf{u}) \Pi n_{\nu}^{2} I_{\nu}^{\circ}\left[T_{i}(s)\right]\right)\left[1-G_{e x t \nu}^{S}\left(\mathbf{u}, s^{\prime}-s\right)\right] .
$$

Indeed, $\mathrm{d} P_{a \nu}^{S}(\mathbf{u}, 0) / \mathrm{d} s$ is equal to $\kappa_{\nu o t}^{S}(\mathbf{u})$, absorption coefficient at the optically thin limit and $C\left(\mathbf{u}, s^{\prime}-s\right) \kappa_{\nu \text { ot }}^{S}(\mathbf{u}) \Pi n_{\nu}^{2} I_{\nu}^{\circ}\left[T_{i}(s)\right]$ can be considered as the emission source term seen from the precise point $s^{\prime}$, which strongly depends on $s^{\prime}-s$ !

Note that $C\left(\mathbf{u}, s^{\prime}-s\right)$ commonly varies[11] between 0.5 and 1.5. The correlation coefficient is easily determined within the statistical Monte Carlo method in use for obtaining the statistical radiative properties of the phase.

The assumption of Taine et al.[12, 7], which uses an emission source term based on the generalised absorption coefficient at equilibrium, is only valid at the limit of a locally optically thick medium. Indeed, the generalised absorption coefficient at equilibrium $K_{\nu}(\mathbf{u})$ is equal to $C(\mathbf{u}, \infty) \kappa_{\nu \text { ot }}^{S}(\mathbf{u})$. Note that the application of the work of Chahlafi et al.[7], based on the previous model, is limited to the determination of a radiative conductivity tensor and more generally to radiative transfer, for a locally optically thick medium: The used assumption is then valid.

Another consequence of the present study is that the GRTE cannot be expressed in a differential formulation as in Ref.[12] but only in integral formulation, i.e.

$$
\begin{aligned}
I_{\nu a}\left(\mathbf{u}, s^{\prime}\right) & =\kappa_{\nu t}^{S}(\mathbf{u}) \Pi n_{\nu}^{2} I_{\nu}^{\circ}\left[T_{i}\left(s^{\prime}\right)\right]+S_{s c \nu a}^{S}\left(\mathbf{u}, s^{\prime}\right) \\
& +\int_{s_{b}}^{s^{\prime}}\left(\overline{S_{e \nu}^{S}(\mathbf{u}, s) \tau_{\nu}\left(\mathbf{u}, s^{\prime}-s\right)}+S_{s c \nu}^{S}(\mathbf{u}, s)\left[1-G_{e x t \nu}^{S}\left(\mathbf{u}, s^{\prime}-s\right)\right]\right) \mathrm{d} s \\
& +\Pi I_{\nu}\left(\mathbf{u}, s_{w}\right)\left[1-G_{e x t \nu}^{S}\left(\mathbf{u}, s^{\prime}-s_{w}\right)\right]
\end{aligned}
$$

Remember that, in a non Beerian homogenised phase, the intensity is only a local property, which does not allow its variation to be simply determined[12]. Equation 18 is only valid at the point $M^{\prime}\left(s^{\prime}\right)$; In particular, its emission term $\kappa_{\nu O T}^{S}(\mathbf{u}) \Pi n_{\nu}^{2} I_{\nu}^{\circ}\left[T_{i}\left(s^{\prime}\right)\right]$ has to be correlated to any extinction phenomenon. 


\section{Validity and limitations of previous studies}

In the works of Tancrez and Taine[1], Bellet et al.[4], Chalhafi et al.[7], for OT or OST cases, and Zeghondy et al.[2] for a STT case, statistically homogeneous media have been characterised by extinction cumulative distribution functions $G_{e x t}$ and phase functions $p$ issued from random points of the volume of the propagation phase, instead of random interfacial points, in principle required for determining $G_{e x t}^{S}$ and $p^{S}$. On the other hand, remember that the direct determination of $G_{e x t}$ is easier and more accurate than the one of $G_{\text {ext }}^{S}$. When $G_{\text {ext }}$ and $G_{e x t}^{S}$ are equal, it is then more pertinent to determine $G_{\text {ext }}$. Moreover, emission is based on an absorption coefficient in all these works.

In the first paper[1], models of statistically homogeneous and isotropic porous media with an opaque phase and a transparent one have been developed: Sets of Dispersed Overlapping Opaque Spheres within a transparent medium (DOOS) and sets of Dispersed Overlapping Transparent Spheres within an opaque medium. As models for a non Beerian homogenised phase did not exist at that time, the aim was first to check the validity of the Beerian assumption and only in this case to characterise the medium by Beerian effective properties by the RDFI method[1, 2]: Extinction, scattering and absorption coefficients. In the case of DOOS, the homogenised phase is rigorously Beerian: Emission is then exactly characterised by an absorption coefficient and it is rigorous to use $G_{e x t}$ instead of $G_{e x t}^{S}$ for both a diffuse and a Fresnel's reflection law at local scale, as discussed in Sec.2.3. DOTS are approximately Beerian for a porosity of the transparent phase larger than typically 0.6 and the same conclusions can be drawn, as a good approximation. But, for a porosity smaller than the criterion, the homogenised phase is non Beerian: The cumulative distribution function $G_{e x t}$ determined in these conditions is not valid. $G_{e x t}^{S}, p^{S}$ and an emission model based on the reciprocity principle should then be used in the general case. Bellet et al. [4] have assumed that the transparent homogenised phase between regular configurations of opaque diffuse parallel rods is Beerian and determined $G_{\text {ext }}$ and $p^{S}$ and characterised emission with an absorption coefficient: It is consistent. Nevertheless, Chalhafi et al.[7] have shown by a more precise approach that this phase is 
strongly non Beerian. As this last model is only applied in the conditions of validity of the radiative Fourier's law[12, 13], the GRTE degenerates into a classical Beerian radiative transfer equation: The use of $G_{e x t}$ instead of $G_{e x t}^{S}$ and of a generalised absorption coefficient at equilibrium for modeling interfacial emission is then pertinent. In the case of the work of Zeghondy et al.[2] applied to a real mullite foam, of STT type, the globally homogenised medium is also Beerian with a excellent approximation: It justifies the use of $G_{e x t}$ and $p$ for the characterisation of internal and external scattering.

In conclusion, the models developed in these four previous studies are consistent, even if the approximations in use have not always been justified by their authors. Zarrouati et al.[11] have for the first time used the reciprocity principle for determining emission properties from absorption ones in a statistically non homogeneous and anisotropic porous medium, strongly non Beerian of OT type. In this approach, absorption is characterised by impacts over interfacial elements. Consequently it is also the case for modeling emission.

A non Beerian homogenised phase of OT type has been characterised in the statistical model of Taine et al.[12] by a Generalised Radiative Transfer Equation (GRTE) expressed in differential formulation. But, for OT and OST cases, emission cannot be characterised by a generalised absorption coefficient in the general case, as shown in Sec.4 : The GRTE has then to be expressed in the integral formulation given by Eq. 18. Nevertheless, for STT and ST2 cases, a differential formulation of the GRTEs is valid, as there is no interfacial emission.

\section{Conclusion}

When the homogenised propagation phase of a porous medium with real (semi) transparent and opaque phases type is Beerian, quasi Beerian, or non Beerian but verifying the validity conditions of the radiative Fourier's law, the simplest and most accurate approach is based on the use of an extinction cumulative distribution function $G_{e x t}$, scattering and absorption cumulative probabilities $P_{s c}$ and $P_{a}$, and a general phase function $p_{\nu}$ associated with volume isotropic source points, as in $\operatorname{Refs}[1,2,4,7]$. On the contrary, a non Beerian homogenised phase considered in the general case 
has to be modeled by an extinction cumulative distribution function $G_{\text {ext }}^{S}$, scattering and absorption cumulative probabilities $P_{s c}^{S}$ and $P_{a}^{S}$, and a general phase function $p_{\nu}^{S}$ associated with interfacial points, as in Ref.[9] dedicated to a medium with semi transparent and transparent phases. In the case of a diffuse reflection law at local scale $G_{e x t}^{S}$ is simply proportional to the derivative of $G_{e x t}$.

Emission by opaque interfaces is simply modeled with an absorption coefficient, characterising the absorption cumulative probabilities $P_{a}$ for a Beerian or quasi Beerian homogenised phase[1, 4], or with a generalised absorption coefficient when the validity conditions of the radiative Fourier's law are verified for a non Beerian homogenised phase[7]. But, in the general case, interfacial emission by a non Beerian homogenised phase has to be modeled from the reciprocity theorem, as done in Ref.[11]. In this case radiation transfer is modeled from a Generalised Radiative Transfer Equation (GRTE) expressed in integral formulation, introduced in the present work.

\section{Acknowledgement}

The authors warmly thank Prof. Dominique Baillis of LaMCoS, INSA de Lyon, for helpful discussions.

\section{Appendix A. Distribution function of the scattered directions}

The successive sets of interfacial scattering events are easily simulated within a model of the real medium by a statistical Monte Carlo simulation technique. This approach is based, as detailed in this paper, on a correlated treatment of emission or scattering source terms with transmission and extinction by absorption or scattering, but on a non correlation between extinction by scattering and the associated scattering source terms.

Consider, for the sake of simplicity, the case of interfacial emission in OT case. A huge number of rays are shot (emitted) in random directions $\mathbf{u}^{(0)}$ from random interfacial points $M_{0}\left(\mathbf{r}_{0}\right)$ of a shooting zone towards the real transparent phase with the weight $w=\alpha_{\nu}\left[\mathbf{u}^{(0)} \cdot \mathbf{n}\left(\mathbf{r}_{0}\right)\right]$, associated with the interfacial emissivity. A part of these rays, which is not absorbed at the interfaces, is reflected within the phase. 
All the corresponding impact and source points $M_{1}\left(\mathbf{r}_{1}\right)$ become internal scattering source points associated with the first set of scattering events (first reflection in the real medium). The weight $w_{\mathbf{u}^{(0)}}\left(\mathbf{r}_{0}, \mathbf{r}_{1}, \mathbf{u}^{(0)}\right)$ of a given shot from $M_{0}$ to $M_{1}$ in the direction $\mathbf{u}^{(0)}$ is the product of the initial emission weight by the reflection weight, i.e.

$$
w_{\mathbf{u}^{(0)}}\left(\mathbf{r}_{0}, \mathbf{r}_{1}, \mathbf{u}^{(0)}\right)=\alpha_{\nu}\left[\mathbf{u}^{(0)} \cdot \mathbf{n}\left(\mathbf{r}_{0}\right)\right]\left(1-\alpha_{\nu}\left[-\mathbf{u}^{(0)} \cdot \mathbf{n}\left(\mathbf{r}_{1}\right)\right]\right),
$$

if $\mathbf{r}_{0}$ is the corresponding initial point. The weight of the point $M_{1}$ as source point in the direction $\mathbf{u}^{(1)}$ is then

$$
W_{\mathbf{u}^{(1)}}^{(1)}\left(\mathbf{r}_{1}\right)=\frac{\int_{\Sigma / V / \mathbf{u}_{1}^{(0)} \cdot \mathbf{n}\left(\mathbf{r}_{0}\right)>0} \int_{0}^{4 \pi} w_{\mathbf{u}^{(0)}}\left(\mathbf{r}_{0}, \mathbf{r}_{1}, \mathbf{u}^{(0)}\right) \delta\left(\mathbf{u}^{(1)}-\mathbf{u}_{1}^{(0) *}\right) \mathrm{d} \Omega_{1}\left(\mathbf{u}^{(0)}\right) \mathrm{d} \mathbf{r}_{0}}{\int_{\Sigma / V / \mathbf{u}_{1}^{(0)} \cdot \mathbf{n}\left(\mathbf{r}_{0}\right)>0} \int_{0}^{4 \pi} w_{\mathbf{u}^{(0)}}\left(\mathbf{r}_{0}, \mathbf{r}_{1}, \mathbf{u}^{(0)}\right) \mathrm{d} \Omega_{1}\left(\mathbf{u}^{(0)}\right) \mathrm{d} \mathbf{r}_{0}},
$$

where $\mathbf{u}_{1}^{(0) *}$ is the conjugate direction of $\mathbf{u}^{(0)}$ by the Fresnel's law. The interfacial distribution function of the scattered directions $\mathbf{u}^{(1)}$, defined in the fixed frame, $f_{\mathbf{u}^{(1)}}^{S(1)}\left(\mathbf{r}_{1}\right)$ then writes

$$
f_{\mathbf{u}^{(1)}}^{S(1)}\left(\mathbf{r}_{1}\right)=\frac{W_{\mathbf{u}^{(1)}}^{(1)}\left(\mathbf{r}_{1}\right)}{\int_{\Sigma_{\mathbf{u}^{(1)}}^{(1)} / V / \mathbf{u}^{(1)} \cdot \mathbf{n}\left(\mathbf{r}_{1}^{\prime}\right) \geq 0} W_{\mathbf{u}^{(1)}}^{(1)}\left(\mathbf{r}_{1}^{\prime}\right) \mathrm{d} \mathbf{r}_{1}^{\prime}} .
$$

In a similar manner, the weight $w_{\mathbf{u}^{(1)}}\left(\mathbf{r}_{1}, \mathbf{r}_{2}, \mathbf{u}^{(1)}\right)$ of a given shot from $M_{1}$ to $M_{2}$ in the direction $\mathbf{u}^{(1)}$ is the product of $W_{\mathbf{u}^{(1)}}^{(1)}\left(\mathbf{r}_{1}\right)$ by the weight associated with the second reflection, i.e.

$$
w_{\mathbf{u}^{(1)}}\left(\mathbf{r}_{1}, \mathbf{r}_{2}, \mathbf{u}_{1}^{(1)}\right)=W_{\mathbf{u}^{(1)}}^{(1)}\left(\mathbf{r}_{1}\right)\left(1-\alpha_{\nu}\left[\mathbf{u}^{(1)} \cdot \mathbf{n}\left(\mathbf{r}_{2}\right)\right]\right)
$$

Equations similar to A.2 and A.3 are then used for obtaining $W_{\mathbf{u}^{(2)}}^{(2)}\left(\mathbf{r}_{2}\right)$ and $f_{\mathbf{u}^{(2)}}^{S(2)}\left(\mathbf{r}_{2}\right)$. This last quantity allows $G_{\text {ext }}^{S(2)}\left(\mathbf{u}^{(2)}, s^{\prime}-s\right)$ to be determined by Eq.4. The previous approach is then iterated for obtaining $G_{e x t}^{S(n)}\left(\mathbf{u}^{(n)}, s^{\prime}-s\right)$. Similar approaches are used for determining $G_{e x t}^{\prime S(n)}\left(\mathbf{u}^{(n)}, s^{\prime}-s\right)$. In all cases, the Monte Carlo calculations are stopped when the power bundle is absorbed instead of being internally scattered. 


\section{References}

[1] M. Tancrez and J. Taine. Direct identification of absorption and scattering coefficients and phase function of a porous medium by a Monte Carlo technique. International Journal of Heat and Mass Transfer, 47:373-383, 2004.

[2] B. Zeghondy, E. Iacona, and J. Taine. Determination of the anisotropic radiative properties of a porous material by radiative distribution function identification (RDFI). International Journal of Heat and Mass Transfer, 49:2810-2819, 2006.

[3] J. Petrasch, P. Wyss, and A. Steinfeld. Tomography-based Monte Carlo determination of radiative properties of reticulated porous ceramics. Journal of Quantitative Spectroscopy and Radiative Transfer, 105:180-197, 2007.

[4] F. Bellet, E. Chalopin, F. Fichot, E. Iacona, and J. Taine. RDFI determination of anisotropic and scattering dependent radiative conductivity tensors in porous media: Application to rod bundles. International Journal of Heat and Mass Transfer, 52:1544-1551, 2009.

[5] S. Haussener, P. Coray, W. Lipinski, P. Wyss, and A. Steinfeld. Tomographybased heat and mass transfer characterization of reticulate porous ceramics for high-temperature processing. J. of Heat Transfer, 132(023305):1-9, 2010.

[6] S. Haussener, W. Lipinski, P. Wyss, and A. Steinfeld. Tomography-based analysis of radiative transfer in reacting packed beds undergoing a solid-gas thermochemical transformation. J. of Heat Transfer, 132(061201):1-7, 2010.

[7] M. Chahlafi, F. Bellet, F. Fichot, and J. Taine. Radiative transfer within non Beerian porous media with semitransparent and opaque phases in non equilibrium: Application to reflooding of a nuclear reactor. Int. J. Heat Mass Transfer, 55(13-14):3666-3676, 2012.

[8] S. Haussener, W. Lipinski, J. Petrasch, P. Wyss, and A. Steinfeld. Tomographic Characterization of a Semitransparent-Particle Packed Bed and Determination of its Thermal Radiative Properties. J. Heat Transfer, 131(072701):1-7, 2009. 
[9] Y. Dauvois. PhD thesis, Ecole Centrale Paris, Grande Voie desVignes, Chatenay Malabry, 92295 cedex, France., 2016.

[10] M. Loretz, E. Maire, and D. Baillis. Analytical modelling of the radiative properties of metalic foams: contribution of x-ray tomography. Advanced Engineering Materials, 10:352-360, 2008.

[11] M. Zarrouati, F. Enguehard, and J Taine. Radiative transfer within strongly non homogeneous porous media: Application to a slab of packed particles. International Journal of Heat and Mass Transfer, 91:936-947, 2015.

[12] J. Taine, F. Bellet, V. Leroy, and E. Iacona. Generalized radiative transfer equation for porous medium upscaling: Application to the radiative Fourier law. Int. J. Heat Mass Transfer, 53(19-20):4071-4081, 2010.

[13] H. Gomart and J. Taine. Validity criterion of the radiative Fourier law for an absorbing and scattering medium. Physical Review E, 83(2):1-8, 2011.

[14] J. Howell, R. Siegel, and P. Menguc. Thermal Radiation Heat Transfer. CRC Press, Washington, 5th edition, 2011.

[15] M. Modest. Radiative Heat Transfer. Academic Press, Inc., 2013.

[16] J. Taine, F. Enguehard, and E. Iacona. Transferts Thermiques. Dunod, Paris, $5^{\text {th }}$ edition, 2014. 